\title{
Immunohistological studies of the kidney in systemic lupus erythematosus and systemic sclerosis using antisera to IgG, C3, fibrin, and human renal glomeruli
}

\author{
D. G. SCOTT AND N. R. ROWELL \\ From The University Departments of Immunology and Dermatology, The General Infirmary at Leeds
}

It is now accepted that systemic lupus erythematosus (SLE) is an autoimmune disease. The view that autoimmunity plays a role in the pathogenesis of systemic sclerosis (SS) is based upon (i) the demonstration that isologous pro-collagen is antigenic in guinea pigs (Steffen, 1965), (ii) the similarity between the skin changes of homologous disease in rats and those in SS (Stastny, Stembridge, and Ziff, 1963), and (iii) the frequent occurrence of antinuclear antibodies in the serum of patients with SS (Beck, Anderson, Gray, and Rowell, 1963; Jordon, Deheer, Schroeter, and Winkelmann, 1971). More direct evidence for the participation of immune mechanisms in the pathogenesis of SS has been provided by Schäfer and Schäfer (1968), who found that 7S $\gamma$-globulin may occur in the fibrinoid material in the kidney in SS.

The present study was undertaken to determine whether further direct evidence for the role of immunity in SS could be obtained by using antisera against human renal glomeruli as well as against IgG, $\mathrm{C} 3$, and fibrin. The findings were compared with the results of similar studies of kidneys from patients with SLE.

Antisera against glomerular capillary basement membranes (GBM) have been used by Nagasawa and Shibata (1970) in immunofluorescence studies of a variety of renal diseases. However, attempts to relate changes found using these GBM antisera to the deposition of immunoglobulins were described only for diabetic glomerulosclerosis.

\section{Material and methods}

\section{CLINICAL MATERIAL}

Samples of kidney were obtained at autopsy from eight patients with SLE and at autopsy or biopsy from nine patients with SS. The clinical and biochemical details of the SLE group of patients are summarized in Table I and of the SS group in Table II. Five of the SLE group of patients and six of the patients with SS had presented evidence of renal involvement during life.
PREPARATION AND STAINING OF SECTIONS Unfixed blocks of kidney were frozen in liquid nitrogen and sectioned in a cryostat. Sections were either (a) washed for 45 to 60 mins in 3 changes of phosphate buffered saline $\left(0.05 \mathrm{~mol} / 1 . \mathrm{PO}_{4}, \mathrm{pH} 8.6\right)$, fixed in $95 \%$ ethyl alcohol at room temperature and dried at $37^{\circ} \mathrm{C}$, or (b) left untreated.

Washed fixed sections were screened for immunoglobulins using polyvalent antihuman immunoglobulin (Ig) antisera labelled with fluorescein isothiocyanate (FITC). Where indicated, adjacent sections were stained for IgG and C3. Staining for fibrin was also carried out in eight cases. Unwashed, unfixed sections were reacted with FITC-labelled antihuman glomerulus antisera.

Standard methods for direct immunofluorescence (Nairn, 1969) were used for the detection of IgG and fibrin and for the staining of sections with FITC-labelled antiglomerulus antisera. The indirect procedure was used for the detection of $\mathrm{C} 3$. Methods used in the examination and photography of sections have been described previously (Scott, 1957; Scott and Rowell, 1965).

Commercial FITC-labelled antisera to human IgG (Burroughs Wellcome) and to fibrin (Nordic Diagnostics) were used. They were used at dilutions which produced no nonspecific staining of tissues; anti-IgG $1: 32$, antifibrin $1: 6$. The lack of cross reactivity of the labelled anti-IgG and antifibrin antisera in staining experiments was checked by blocking experiments using unlabelled anti-IgG and antifibrin antisera. The lack of cross reactivity of these unlabelled antisera was checked by immunoelectrophoresis.

An unlabelled goat antihuman $\beta_{1} \mathrm{C} / \beta_{1} \mathrm{~A}$ antiserum (Nordic Diagnostics) and an FITC-labelled rabbit antiserum to goat immunoglobulin (RAG/FITC) were used for the detection of $\mathrm{C} 3$ by the indirect procedure. The specificity of the anti- $\beta_{1} \mathrm{C}$ antiserum and its lack of cross reactivity with immunoglobulins, were checked by immunoelectrophoresis. The RAG/FITC antiserum was absorbed with a crude globulin fraction of human serum and with a homogenate of human renal glomeruli before use in staining experiments.

Methods used in the raising of the antihuman glomerulus antisera, their labelling with FITC, and their absorption with human $\mathrm{AB}$ red cells and normal human serum and in the study of their antitissue specificity, have been described previously (Scott and Rowell, 1967). 
Table I Patients with SLE

\begin{tabular}{|c|c|c|c|c|c|c|c|c|}
\hline $\begin{array}{l}\text { Case } \\
\text { no. }\end{array}$ & $\begin{array}{l}\text { Age } \\
\text { and } \\
\text { sex }\end{array}$ & $\begin{array}{l}\text { Duration } \\
\text { of } \\
\text { disease } \\
\text { (yrs) }\end{array}$ & Main features & $\begin{array}{l}\text { Blood } \\
\text { pressure }\end{array}$ & $\begin{array}{l}\text { Creatinine } \\
\text { clearance } \\
(\mathrm{ml} / \mathrm{min})\end{array}$ & $\begin{array}{l}\text { Blood } \\
\text { urea } \\
(\mathrm{mgl} \\
100 \mathrm{ml})\end{array}$ & $\begin{array}{l}\text { Albumin- } \\
\text { uria }\end{array}$ & Cause of death \\
\hline 1 & $\begin{array}{l}25 \\
F\end{array}$ & $5 \frac{1}{2}$ & $\begin{array}{l}\text { Rash, alopecia, intermittent } \\
\text { fever, hypertension, } \\
\text { anaemia, oedema }\end{array}$ & $180 / 130$ & & 96 & + & $\begin{array}{l}\text { Uraemia, } \\
\text { pneumonia }\end{array}$ \\
\hline 2 & $\mathbf{F}$ & 9 & $\begin{array}{l}\text { Rash, alopecia, purpura, } \\
\text { hypertension }\end{array}$ & $210 / 120$ & & 13 & + & $\begin{array}{l}\text { Subarachnoid } \\
\text { haemorrhage }\end{array}$ \\
\hline 3 & $\begin{array}{l}47 \\
\mathbf{M}\end{array}$ & 13 & $\begin{array}{l}\text { Rash, alopecia, cutaneous } \\
\text { vasculitis }\end{array}$ & $150 / 90$ & & & & Pneumonia \\
\hline 4 & 53 & 4 & $\begin{array}{l}\text { Rash, alopecia, joint pains, } \\
\text { psychosis }\end{array}$ & $130 / 80$ & 180 & 29 & $\operatorname{Tr}$ & $\begin{array}{l}\text { Acute pleurisy, } \\
\text { mediastinitis, } \\
\text { myocarditis }\end{array}$ \\
\hline 5 & $\begin{array}{l}20 \\
\mathbf{M}\end{array}$ & 2 & $\begin{array}{l}\text { Rash, joint pains, nephrotic } \\
\text { syndrome }\end{array}$ & $160 / 90$ & 63 & 516 & + & Uraemia \\
\hline 6 & $\stackrel{49}{\mathrm{~F}}$ & 3 & $\begin{array}{l}\text { Haemolytic anaemia, fever, } \\
\text { splenomegaly, gravita- } \\
\text { tional oedema, leg ulcers }\end{array}$ & $130 / 80$ & 71 & 37 & - & $\begin{array}{l}\text { (Specimen taken } \\
\text { at renal } \\
\text { biopsy) }\end{array}$ \\
\hline 7 & $\begin{array}{l}22 \\
\mathbf{F}\end{array}$ & 2 & $\begin{array}{l}\text { Raynaud's phenomenon, } \\
\text { joint pains, epilepsy, } \\
\text { anaemia, hypertension }\end{array}$ & $195 / 90$ & & 275 & + & Uraemia \\
\hline 8 & $\begin{array}{l}37 \\
\mathbf{F}\end{array}$ & 18 & $\begin{array}{l}\text { Rash, Raynaud's pheno- } \\
\text { menon, arthritis, } \\
\text { pericarditis }\end{array}$ & $140 / 90$ & & & + & Pneumonia \\
\hline
\end{tabular}

\section{Observations}

\section{WITH ANTIGLOMERULUS ANTISERUM}

Appearances seen in fluorescein-antiglomerulus stained sections of kidney from patients with SLE were similar to those seen in SS but differed in a number of ways from those seen in normal kidneys.

\section{In renal glomeruli}

In the normal renal glomerulus fluorescein-antiglomerulus conjugates produced fine linear staining of capillary loops and the inner aspect of Bowman's membrane (Fig. 1).

Glomerular changes in both SLE and SS comprised focal or diffuse thickening of capillary basement membranes (Fig. 2), and the appearance of filmy or fibrillar intercapillary staining (Fig. 3). In glomeruli undergoing sclerosis there was partial or complete replacement of the tuft by green fibrillar staining or blue collagen-like autofluorescence.

\section{In arteries and arterioles}

The tunica media of normal renal arteries and arterioles stained by fluorescein-antiglomerulus conjugates showed a fine reticulate pattern and the internal elastic lamina only autofluorescence. Neither staining nor autofluorescence was seen in the intima.
Changes seen in arterioles in both SLE and SS consisted of smudging of medial reticulum (Fig. 3) or the replacement of the normal reticulate pattern of medial staining by homogeneous staining or by blue autofluorescence (Fig. 4). Changes seen in larger arteries included green fibrillar staining or blue collagen-like autofluorescence of the intima and the appearance of a fibrillar, rather than reticulate, pattern of medial staining. Smudging of medial reticulum or the partial or complete replacement of medial staining by collagen-like autofluorescence was also seen (Table III).

RELATION BETWEEN ALTERATIONS IN DISTRIBUTION OF GLOMERULAR AND ARTERIOLAR ANTIGENS AND DEPOSITION OF IgG OR FIBRIN

\section{In SLE}

In SLE abnormalities shown by antiglomerulus antisera tended to be in those areas where $\mathrm{IgG}$, or IgG and $\mathrm{C} 3$ was also demonstrable.

Broadening of glomerular capillary basement membranes was found in 5 kidneys and in each case granular staining or granular plus linear staining for IgG (Fig. 5) was found in adjacent sections. When IgG was found in glomerular capillary basement membranes it was usually present in the mesangium 


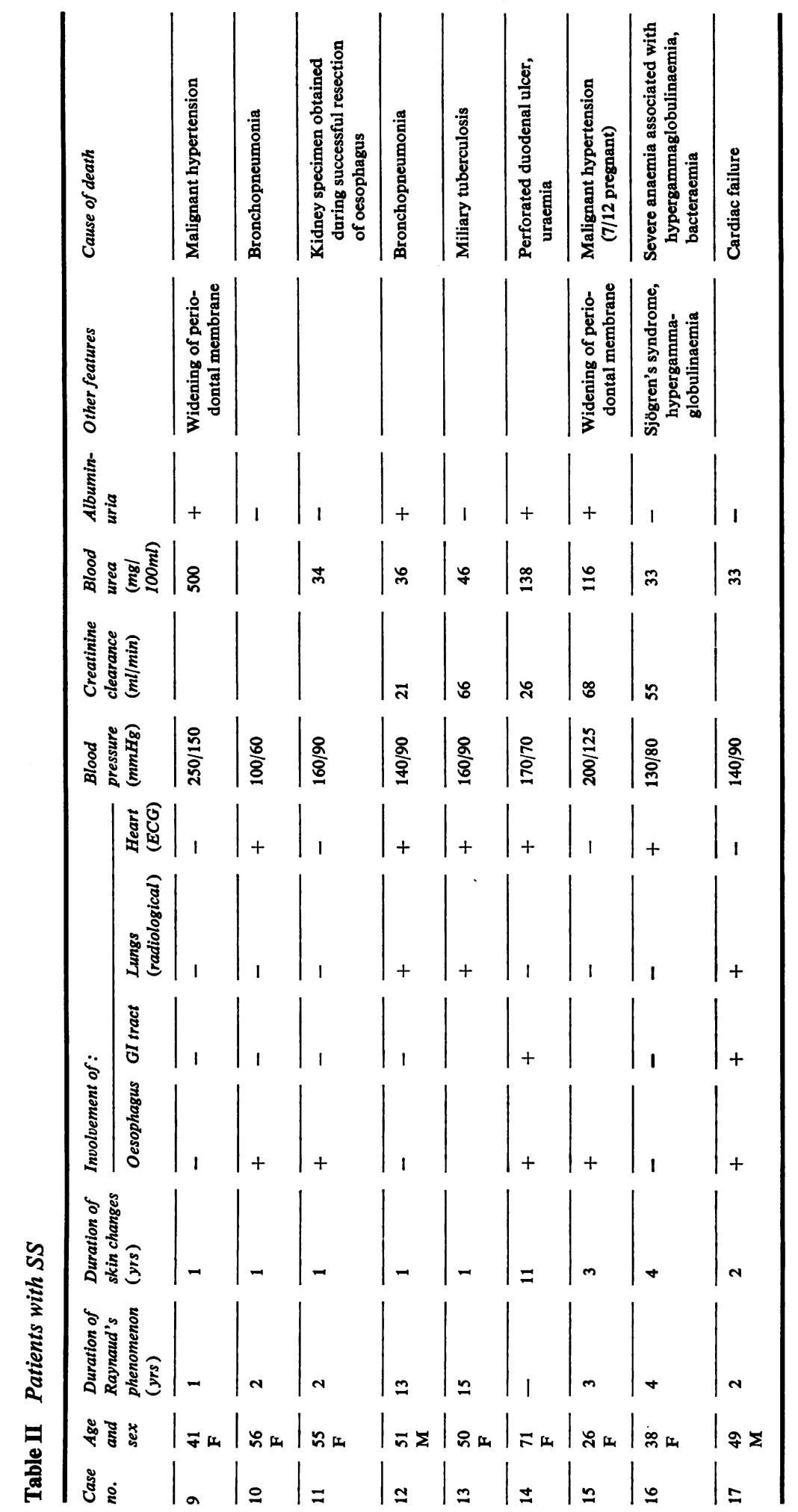




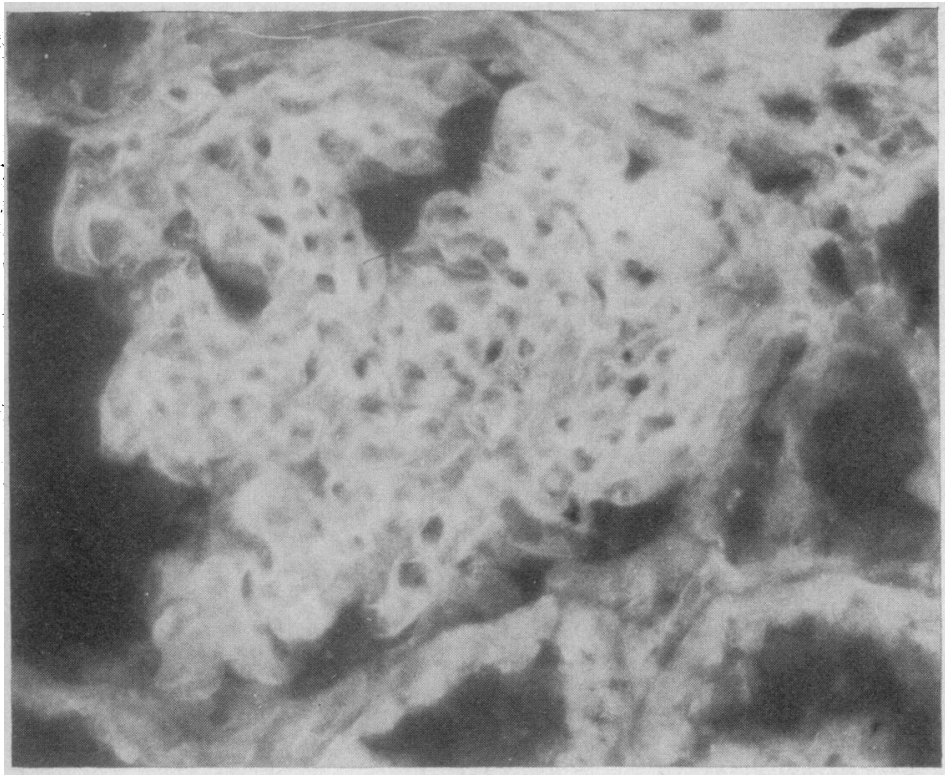

FIG. 1 Normal human glomerulus stained with the fluorescein-antiglomerulus conjugate. The capillary basement membranes show uniform linear staining. Original magnification $\times 100$

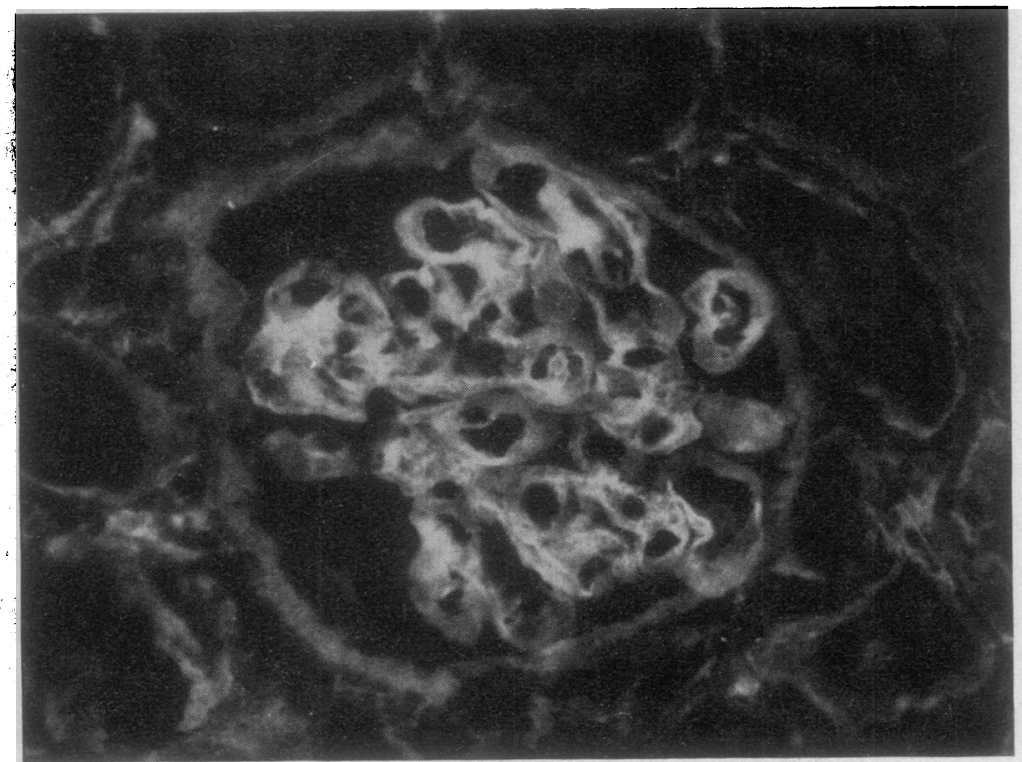

FIG. 2 Renal glomerulus in systemic sclerosis (Case 17) stained with the fluoresceinantiglomerulus conjugate. There is focal thickening of capillary basement membranes. Original magnification $\times 80$

also (Table III). In two cases IgG was found only in the mesangium and, in a third case, glomeruli showed no staining for IgG. FITC-antiglomerulus stained sections from these kidneys showed little or no broadening of glomerular capillary basement membranes.

Alterations in the pattern of staining of arteriolar media-smudging of medial reticulum or homogeneous staining of the media-were found in FITC-antiglomerulus stained sections from 5 SLE kidneys. Deposits of IgG or of IgG and C3 were found in the walls of arterioles in 4 of these kidneys.
The presence of intramural deposits of IgG and C3 in the absence of arteriolar changes in FITC-antiglomerulus stained sections was shown in one instance (Case 4, Table III).

In $S S$

In general the association between FITC-antiglomerulus changes and IgG and $\mathrm{C} 3$ was less marked in SS than in SLE.

In SS, broadening of glomerular capillary basement membranes was seen in FITC-antiglomerulus stained sections from 5 kidneys. In 1 of these kidneys IgG 


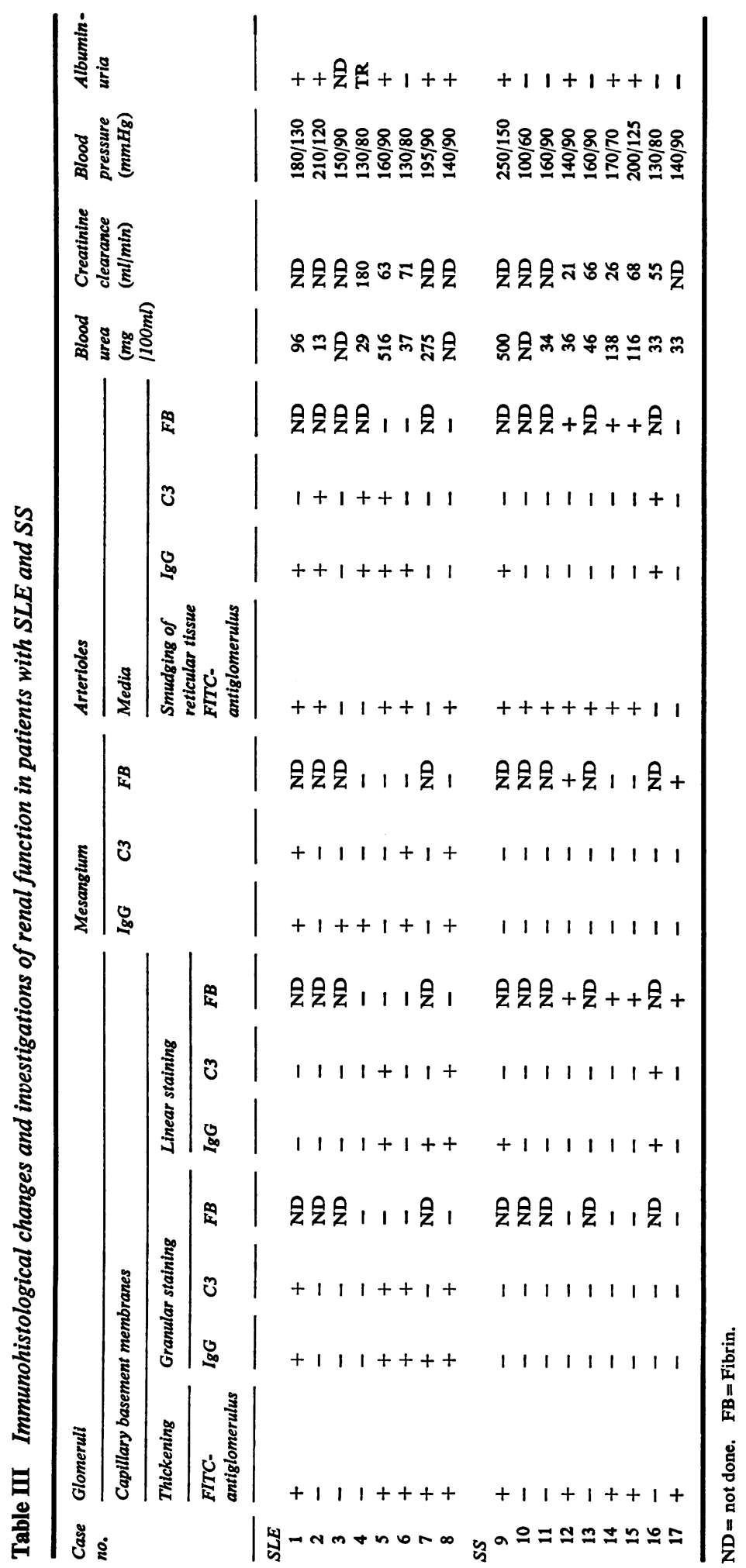




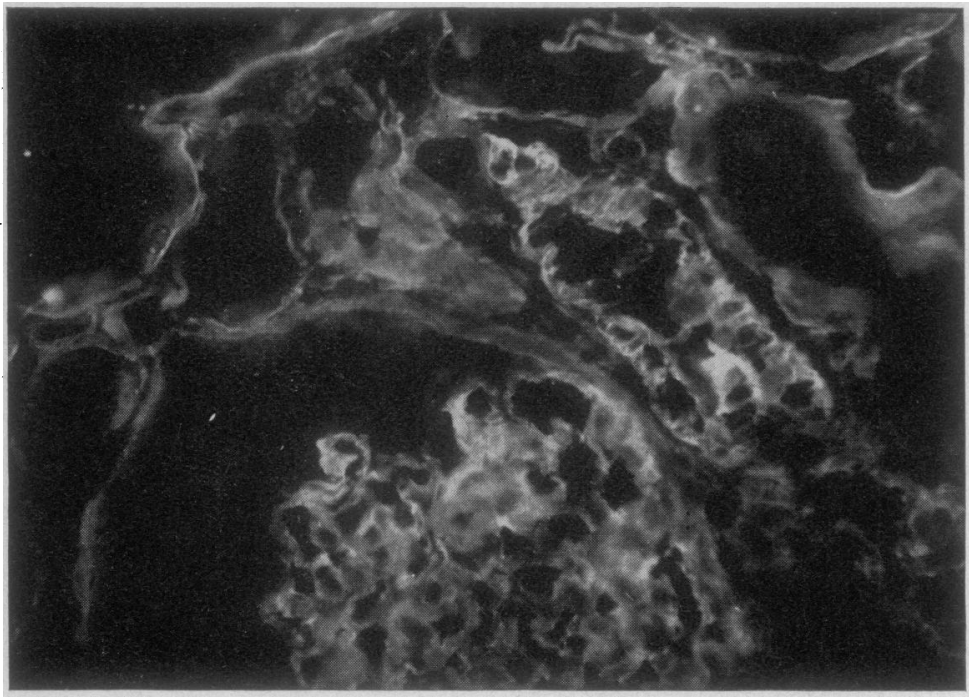

FIG. 3 Systemic sclerosis kidney (Case 14) stained with the fluorescein-antiglomerulus conjugate. The glomerular capillaries show focal is thickening. There is some filmy intercapillary staining and also. smudging of the reticulum in the media of the arteriole. Original magnification $\times 100$

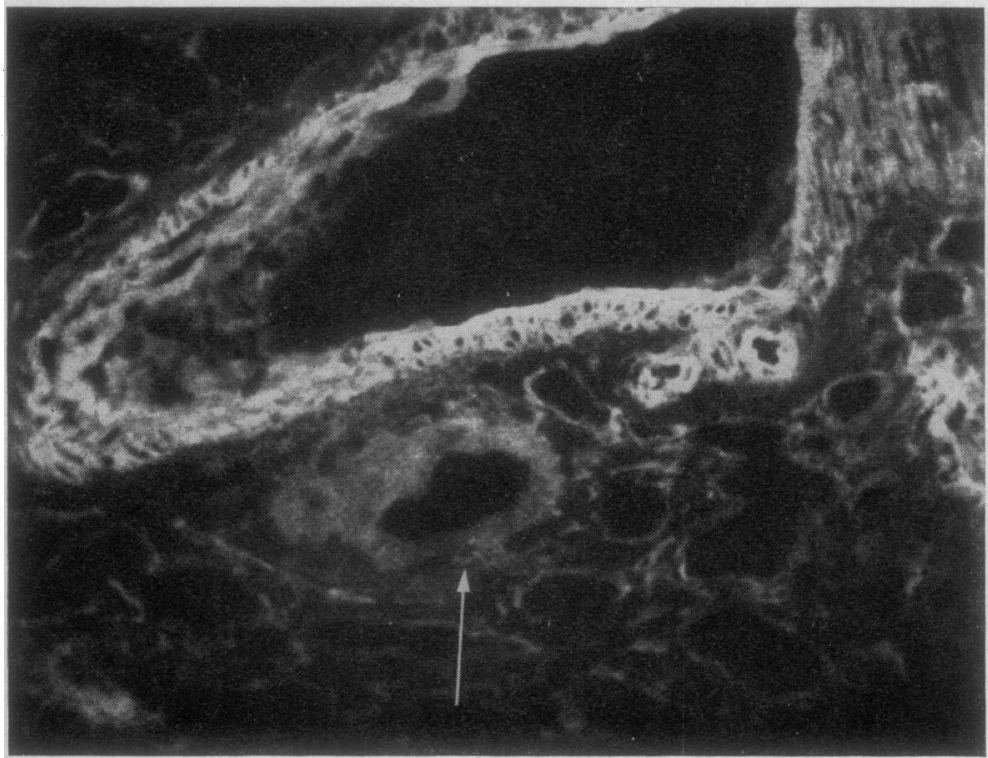

FIG. 4 Blood vessels in system sclerosis kidney (Case 15) stainea with the fluorescein-antiglomerulus conjugate. A small artery (arrowed) showing dull homogeneous staining of its tunica media lies below an essentially normal large artery. The small arterioles to the right show smudgy staining in their walls. Original magnification $\times 60$

was found as a smooth linear deposit on glomerular capillary basement membranes. The presence of smooth linear staining of glomerular capillaries for IgG and $\mathrm{C} 3$ in the absence of broadening of glomerular capillary basement membranes was seen in one instance.

Arteriolar changes similar to those found in SLE were seen in FITC-antiglomerulus stained sections from 7 SS kidneys, but in 6 of these kidneys the arterioles showed no staining for IgG (Table III).

Sections from 4 SS kidneys were stained for fibrin. Fibrin was found in the glomeruli in all of these kidneys and in arterioles in 3 of them. In glomeruli fibrin staining involved segments of capillary loops (Fig. 6) but it was also found between capillaries and on Bowman's membrane. In arterioles staining for fibrin was seen as smudgy green fluorescence in the $\frac{7}{O}$ walls of the vessels, and was also found extravascularly on basement membranes of neighbouring $\stackrel{\sim}{\sim}$ tubules (Fig. 6). Broadening of glomerular capillary $\mathrm{N}$ basement membranes was present in all 4 kidneys $\mathcal{N}$ showing glomerular staining for fibrin, and smudging $\omega$ of the medial reticulum of arterioles was found in the 3 kidneys showing intramural deposits of fibrin.

\section{Discussion}

The antiglomerulus antiserum used in the present studies is known to react with a wide variety of $\frac{\Omega}{\mathbb{D}}$ connective tissue antigens (Hill and Cruickshank, $\frac{0}{0}$ 


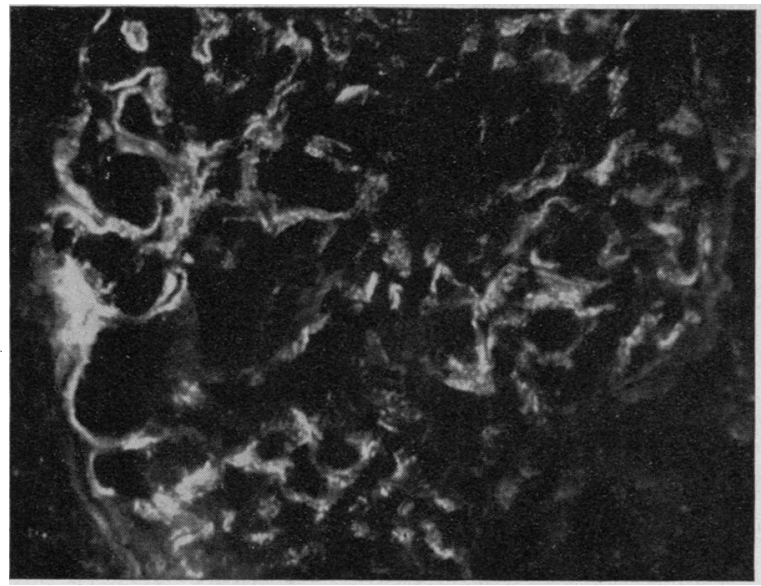

FIG. 5 Case 5. Renal glomerulus in SLE stained for C3. There is linear and granular staining of capillary basement membranes. Granular staining is seen also in the mesangium. Original magnification $\times 100$

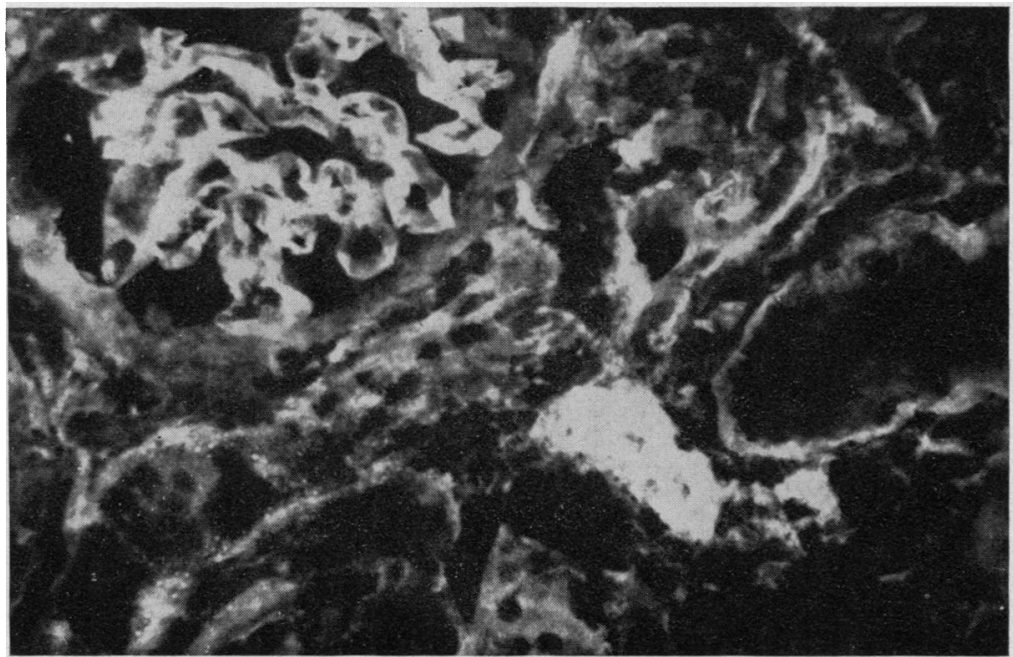

FIG. 6 Same kidney as in Fig. 3 stained for fibrin. Fibrin appears in the wall of arteriole and around the vessel and on the basement membranes of $\vec{\theta}$ renal tubules. Staining of vary천 ing intensity is seen also in the glomerular capillary tuft and appears to involve capillary basement membranes. Original magnification $\times 75$

1953). Thus, the glomerular capillary basement membrane changes we have found using this antiserum could be attributable either to a thickening of the membrane itself or to the appearance of nonbasement membrane antigens in a damaged membrane. Homogeneous thickening of glomerular capillary basement membranes in lupus nephritis has been described by Nagasawa and Shibata (1970) who used fluorescein-labelled antisera against purified basement membrane antigens. This makes it unlikely that the changes occurring in glomerular capillary basement membranes in SLE are to be attributed to the appearance of nonbasement membrane antigens.

We have tried to relate alterations in the distribution of glomerular and arteriolar antigens, revealed by the use of FITC-antihuman glomerulus antisera, to sites of deposition of IgG, C3, and fibrin. In staining experiments with FITC-antiglomerulus antisera, alterations in the pattern of staining in the media of arterioles and broadening of glomerular capillary basement membranes occurred both in SLE and in SS. In SLE these changes appeared to occur in association with the deposition of IgG or of IgG and C3 in the walls of arterioles and on the basement membranes of glomerular capillaries. A similar association between alterations in the distribution of glomerular and arteriolar antigens and the deposition of IgG was not so common in SS.

It is generally agreed that the renal lesions of SLE are attributable to the deposition of immune complexes (Koffler, Schur, and Kunkel, 1967; Schur and Sandson, 1968) and that clinical renal disease is associated with deposits on glomerular capillary basement membranes (Koffler, Agnello, Carr, and Kunkel, 1969). Our observations are in agreement with these views. In SLE material glomerular staining for IgG appeared, in the main, in the form of granules either on capillary basement membranes or in the mesangium, or in both of these sites. Occasionally capillary basement membranes showed mixed linear 
and granular staining. Where IgG was confined to the mesangium there had been no clinical evidence of renal disease during life and little or no broadening of glomerular capillary basement membranes was seen in sections stained with the FITC-antiglomerulus conjugate.

Table III shows that in two patients (Cases 3 and 7) no C3 could be detected in association with glomerular deposits of IgG, and in three cases IgG was found both in glomeruli and arterioles, while $\mathrm{C} 3$ was found only in glomeruli (Cases 1 and 6) or only in arterioles (Case 4). Even the finding that in the 1 kidney $\mathrm{C} 3$ could be detected in association with IgG in glomeruli but not in arterioles, or in arterioles but not in glomeruli, does not necessarily point to differences in the constitution of the complexes concerned. The discrepancy between staining for IgG and C3 may merely reflect differences in the total amount of IgG (and, therefore, of C3) present at the different sites. In favour of this is the fact that in our hands the anti$\beta_{1} \mathrm{C} / \beta_{1} \mathrm{~A}$ antiserum used for the detection of $\mathrm{C} 3$ by the indirect method could not be used at dilutions greater than 1:12, whereas the FITC-anti-IgG antiserum could be used at $1: 128$.

Glomerular deposits of IgG usually presented the granular appearance typical of immune complex deposition (Unanue, Dixon, and Feldman, 1968). In some instances, however, a mixed linear and granular pattern was seen. A linear distribution of immunoglobulins on glomerular capillary basement membranes in SLE has previously been described by Koffler and others (1969), who suggested that this finding pointed to the development of anti-glomerular basement membrane antibodies during the course of the disease. Pertinent here is the report by Landry and Sams (1973) that antiepidermal basement membrane antibodies can be detected in eluates from the skin lesions of SLE.

In SS, alterations in the distribution of glomerular and arteriolar antigens could be related to the deposition of IgG in glomeruli and arterioles in only 1 of the 9 kidneys examined. One other kidney showed staining for IgG and C3 in glomeruli and arterioles but no abnormality was seen in FITC-antiglomerulus stained sections. This patient had had hypergammaglobulinaemia for some years and it is possible that the IgG staining seen in glomeruli and arterioles reflected this.

The lack of relation between alterations in the distribution of glomerular and arteriolar antigens and the deposition of IgG in SS contrasts with the situation in SLE and suggests that the evolution of renal disease in the two conditions involves different mechanisms. A further feature in favour of this was the failure to show fibrin in the kidneys in SLE when it was present in association with other evidence of renal damage in all the SS kidneys examined.

\section{Summary}

Kidneys from nine patients with systemic sclerosis (SS) and eight with systemic lupus erythematosus (SLE) were reacted in immunofluorescence studies with antisera to human renal glomeruli, IgG and C3. Sections of 4 kidneys from each group were reacted with antifibrin antisera. Thickening of glomerular capillary basement membranes and alterations in reticular tissue in the media of arterioles were shown by antiglomerulus antisera in comparable frequency in both groups of diseases. In SLE they occurred in association with deposits of IgG or of IgG and $\mathrm{C3}$ in glomerular capillary basement membranes and in the walls of arterioles. This association was less marked in SS. A relationship between glomerular and arteriolar deposits of fibrin and thickening of glomerular capillary basement membranes or changes in the media of arterioles was demonstrable in all SS kidneys reacted with antifibrin antisera. No fibrin was detected in the SLE material. These observations suggest that classical immune mechanisms are not involved in the evolution of renal lesions in SS.

We wish to thank Mrs. J. O'Malley and Mr. R. A. Forster for their excellent technical assistance.

\section{References}

BeCK, J. S., ANDerson, J. R., GrAy, K. G., AND Rowell, N. R. (1963) Lancet, 2, 1188 (Antinuclear and precipitating autoantibodies in progressive systemic sclerosis)

Hill, A. G. S., AND CRUickshank, B. (1953) Brit. J. exp. Path., 34, 27 (A study of antigenic components of kidney tissue)

Jordon, R. E., Deheer, D., Schroeter, A., And Winkelmann, R. K. (1971) Mayo Clin. Proc., 46, 111 (Antinuclear antibodies: their significance in scleroderma)

Koffler, D., Agnello, V., CARR, R. I., AND KUnKel, H. G. (1969) Amer. J. Path., 56, 305 (Variable patterns of immunoglobulin and complement deposition in the kidneys of patients with systemic lupus erythematosus)

-, SCHUR, P. H., AND KUNKeL, H. G. (1967) J. exp. Med., 126, 607 (Immunological studies concerning the nephritis of systemic lupus erythematosus)

LANDRY, M., AND SAMS, W. M., JR. (1973) J. clin. Invest., 52, 1871 (Systemic lupus erythematosus. Studies of the antibodies bound to skin)

NAGASAWA, T., AND Shibata, S. (1970) Jap. J. exp. Med., 40, 23 (Application of the antibody against soluble GBM substance to the immunofluorescence staining of kidney sections) 
NAIRN, R. C. (1969) In 'Fluorescent Protein Tracing', 3rd ed., pp. 502. Livingstone, Edinburgh and London SCHÄFER, H. E., AND SCHÄFER, A. (1968) Arch. klin. Med., 214, 187 (Die progressive Sklerodermie und die terminale Phase der malignen Hypertonie)

Schur, P. H., AND SANDSON, J. (1968) New Engl. J. Med., 278, 533 (Immunologic factors and clinical activity in systemic lupus erythematosus)

Scort, D. G. (1957) Brit.J. exp. Path., 38, 178 (A study of the antigenicity of basement membrane and reticulin)

-, AND Rowell, N. R. (1965) Brit. J. Derm., 77, 211 (Preliminary investigations of arteritic lesions using fluorescent antibody techniques)

—_- (1967) Ann. rheum. Dis., 26, 10 (Alterations in the antigenic constitution of renal glomerular capillaries accompanying the histological maturation of renal glomeruli in the rat)

StASTNY, P., STEMBRidge, V. A., AND ZifF, M. (1963) J. exp. Med., 118, 635 (Homologous disease in the adult rat, a model for autoimmune disease. 1. General features and cutaneous lesions)

StefFen, C. (1965) Ann. N.Y. Acad. Sci., 124, 570 (Antigenicity and autoantigenicity of collagen)

Unanue, E. R., Dixon, F. J., AND Feldman, J. D. (1968) 'Experimental immunologic diseases of the kidney', in 'Textbook of Immunopathology', ed. P. A. Miescher and H. J. Muller-Eberhard, p. 164. Grune and Stratton, New York 